\title{
CFD analysis of thermodynamic cycles in a pulse tube refrigerator
}

\author{
Ling Chen ${ }^{\mathrm{a}}$, Yu Zhang ${ }^{\mathrm{a}, *}$, Ercang Luo ${ }^{\mathrm{b}}$, Teng $\mathrm{Li}^{\mathrm{a}}$, Xiaolin Wei ${ }^{\mathrm{a}}$ \\ ${ }^{a}$ Key Laboratory of Environmental Mechanics, The Institute of Mechanics, Chinese Academy of Sciences, Beijing 100190, China \\ ${ }^{\mathrm{b}}$ Key Laboratory of Cryogenics, The Technical Institute of Physics and Chemistry, Chinese Academy of Sciences, Beijing 100190, China
}

\section{A R T I C L E I N F O}

Article history:

Received 7 June 2010

Received in revised form 18 August 2010

Accepted 26 August 2010

\section{Keywords:}

Pulse tube refrigerator

CFD

Thermodynamic cycles

\begin{abstract}
A B S T R A C T
The objectives of this paper are to study the thermodynamic cycles in an inertance tube pulse tube refrigerator (ITPTR) by means of CFD method. The simulation results show that gas parcels working in different parts of ITPTR undergo different thermodynamic cycles. The net effects of those thermodynamic cycles are pumping heat from the low temperature part to the high temperature part of the system. The simulation results also show that under different frequencies of piston movement, the gas parcels working in the same part of the system will undergo the same type of thermodynamic cycles. The simulated thermal cycles are compared with those thermodynamic analysis results from a reference. Comparisons show that both CFD simulations and theoretical analysis predict the same type of thermal cycles at the same location. However, only CFD simulation can give the quantitative results, while the thermodynamic analysis is still remaining in quality.
\end{abstract}

(c) 2010 Elsevier Ltd. All rights reserved.

\section{Introduction}

Pulse tube refrigerator (PTR) has many advantages over conventional refrigerators, such as no moving part, low cost, high reliability and less mechanical vibration. Therefore, the PTR is recognized as one of the most promising refrigerators in the future. Since the invention of the basic pulse tube refrigerator (BPTR) in the early 1960s [1], pulse tube refrigerator has experienced several significant structural improvements including the orifice pulse tube refrigerator (OPTR) [2], the double inlet pulse tube refrigerator (DIPTR) [3], the multi-stage pulse tube refrigerator [4] and most recently the inertance tube pulse tube refrigerator (ITPTR) [5]. All of these improvements have led to the technical progress of the PTR, and so far the lowest temperature attained by the pulse tube refrigerator has already reached about $1.3 \mathrm{~K}$ [6].

However, till now, the mechanism of PTR has still not yet been fully understood. Several theories have been developed to explain the refrigeration mechanism of PTRs since their invention. Gifford and Longsworth first explained the refrigeration effect in BPTR by a process named surface heat pumping [7], which is determined by both the thermal interactions between the wall of the BPTR and the properties of the working gas; however, this theory is only suitable for PTRs operating under low frequencies. Peter and Radebaugh [8] assumed that phase shift between pressure and the mass flow rate is the major mechanism for heat transfer in PTRs. In 1990s, Liang et al. [9] proposed thermodynamic non-symmetry

\footnotetext{
* Corresponding author. Tel.: +86 10 82544231; fax: +86 1062561284

E-mail address: zhangyu@imech.ac.cn (Y. Zhang).
}

effect to illustrate the refrigeration in PTRs. This theory points out that when gas flows through a tube half filled with porous material, it will experience strong non-symmetric heat exchange and consequently generate refrigeration. Besides those discussions, thermo-acoustic effect is also introduced to explain the mechanism of PTRs. However, all of these theories have their own limitations and cannot explain the mechanism of the PTRs adequately.

The theories mentioned above fail to give detail information of thermodynamic processes of the working gas in a cycle, which must be important for understanding the mechanism of PTRs. The thermodynamic processes of the working gas in PTR are very different from those described in classical thermodynamic cycles such as an ideal Stirling cycle, in which every portion of the gas is assumed to undergo the exact same thermodynamic cycle. In PTR each small portion of the working gas, viz. a gas parcel, oscillates only in a small part, and the thermal cycles at different parts should be also different [10]. In 1997, Liang [10] proposed a sinusoidal model to analyze the thermodynamic processes of gas parcels oscillating in a regenerator. In his analysis, results show that each gas parcel in the regenerator works as either a heat engine or a refrigerator. But Liang did not attain the transient temperature of the gas parcel and the obtained function of the regenerator is questionable. In 2004, a further theoretical study was conducted by Luo [11] to investigate the thermodynamic cycles in the regenerator. That theory is named as meso-scope thermodynamic theory. With some assumptions and thorough mathematical computation, the thermodynamic cycles of these characteristic gas parcels were attained. However, in Luo's analysis some assumptions such as the working gas has no viscosity, the phase 


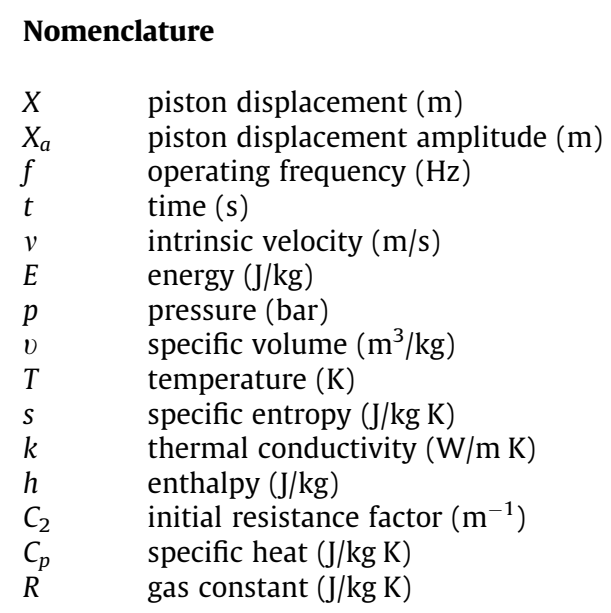

shift between fluctuated pressures and flow rates is keeping uniform everywhere, are not reasonable. Furthermore, those analyses are still qualitative, but have no quantitative meanings.

In this paper, two-dimensional axi-symmetric CFD simulations are conducted to analyze the thermodynamic processes in ITPTR. Instead of assumptions, CFD method directly calculates the gas velocity, the gas temperature, the gas pressure and the phase shift of different variables at different locations. After CFD calculations, summarizing the obtained results in Lagrangian's view, the quantitative results of thermodynamic cycles will come out.

\section{Simulation model}

A two-dimensional, axi-symmetric schematic of the ITPTR system is shown in Fig. 1. The sizes of all parts of the system are listed in Table 1 . The details of the simulated system can be found in the publication of Cha et al. [12].

A commercial CFD code Fluent [14] is applied in this simulation. The amount of computational cells reaches 4600 . The piston movement is described as

$X=X_{a} \sin (\omega t)$

where $X_{a}=4.511 \mathrm{~mm}$ is the amplitude, $\omega=2 \pi f$ is the angular velocity and $f$ is the operating frequency. A User Define Function (UDF) is written to track and guide the movement of the piston.

The mass, momentum, and energy equations solved by Fluent for the working gas are $[12,14]$

$\frac{\partial \rho_{\mathrm{f}}}{\partial t}+\frac{1}{r} \frac{\partial}{\partial r}\left(r \rho_{\mathrm{f}} v_{\mathrm{r}}\right)+\frac{\partial}{\partial x}\left(\rho_{\mathrm{f}} v_{\mathrm{x}}\right)=0$

$\frac{\partial}{\partial t}\left(\rho_{\mathrm{f}} \vec{v}\right)+\nabla \cdot\left(\rho_{\mathrm{f}} \vec{v} \vec{v}\right)=-\nabla p+\nabla \cdot(\overrightarrow{\vec{\tau}})$

\begin{tabular}{|c|c|}
\hline \multicolumn{2}{|c|}{ Greek letters } \\
\hline$\omega$ & angular frequency \\
\hline$\gamma$ & porosity \\
\hline$\rho$ & density $\left(\mathrm{kg} / \mathrm{m}^{3}\right)$ \\
\hline$\alpha$ & permeability \\
\hline$\stackrel{\mu}{\vec{\tau}}$ & $\begin{array}{l}\text { viscosity }(\mathrm{kg} / \mathrm{m} \mathrm{s}) \\
\text { stress tensors }\left(\mathrm{N} / \mathrm{m}^{2}\right)\end{array}$ \\
\hline & \\
\hline $\mathrm{f}$ & fluid \\
\hline $\mathrm{r}$ & radial coordinate \\
\hline S & solid \\
\hline $\begin{array}{l}x \\
\text { ref }\end{array}$ & $\begin{array}{l}\text { axial coordinate } \\
\text { reference }\end{array}$ \\
\hline
\end{tabular}

Table 1

Dimensions of the ITPTR.

\begin{tabular}{lll}
\hline Components & Diameter $(\mathrm{mm})$ & Length $(\mathrm{mm})$ \\
\hline Compressor & 19.08 & 7.5 \\
Transfer line & 3.1 & 101 \\
WHX1 & 8 & 20 \\
Regenerator & 8 & 58 \\
CHX & 6 & 5.7 \\
Pulse tube & 5 & 60 \\
WHX2 & 8 & 10 \\
Inertance tube & 0.85 & 684 \\
Reservoir & 26 & 130 \\
\hline
\end{tabular}

$\frac{\partial}{\partial t}\left(\rho_{\mathrm{f}} E\right)+\nabla \cdot\left(\vec{v}\left(\rho_{\mathrm{f}} E+p\right)\right)=\nabla \cdot\left[k_{\mathrm{f}} \nabla T+(\overrightarrow{\vec{\tau}} \cdot \vec{v})\right]$

where

$E=h-\frac{p}{\rho}+\frac{v^{2}}{2}$

The mass, momentum and energy equations for the porous media are as follows:

$$
\begin{aligned}
& \frac{\partial}{\partial t}\left(\gamma \rho_{\mathrm{f}}\right)+\frac{1}{r} \frac{\partial}{\partial r}\left(\gamma r \rho_{\mathrm{f}} v_{\mathrm{r}}\right)+\frac{\partial}{\partial x}\left(\gamma \rho_{\mathrm{f}} v_{\mathrm{x}}\right)=0 \\
& \frac{\partial}{\partial t}\left(\gamma \rho_{\mathrm{f}} \vec{v}\right)+\nabla \cdot\left(\gamma \rho_{\mathrm{f}} \vec{v} \vec{v}\right)=-\gamma \nabla p+\nabla \cdot(\gamma \vec{\tau}) \\
& -\left(\frac{\mu}{\alpha} \vec{v}+C_{2} \frac{1}{2} \rho_{\mathrm{f}}|\vec{v}| \vec{v}\right) \\
& \frac{\partial}{\partial t}\left[\gamma \rho_{\mathrm{f}} E_{\mathrm{f}}+(1-\gamma) \rho_{\mathrm{s}} E_{\mathrm{s}}\right]+\nabla \cdot\left[\overrightarrow{\mathcal{v}}\left(\rho_{\mathrm{f}} E_{\mathrm{f}}+p\right)\right] \\
& =\nabla \cdot\left[\left(\gamma k_{\mathrm{f}}+(1-\gamma) k_{\mathrm{s}}\right) \nabla T+(\gamma \overrightarrow{\vec{\tau}} \cdot \vec{v})\right]
\end{aligned}
$$

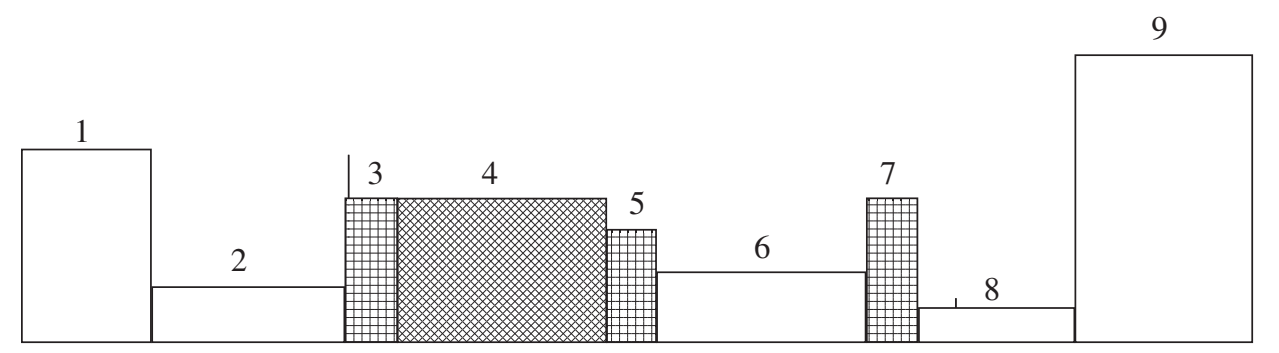

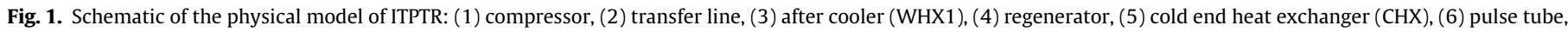
(7) hot end heat exchanger (WHX2), (8) inertance tube, and (9) reservoir. 
where $\gamma$ is porosity of the porous media, $\alpha$ is the permeability and $C_{2}$ is the inertial resistance factor. In simulation, $\gamma=0.69, \alpha=1.06 \times$ $10^{-10} \mathrm{~m}^{2}$, and $C_{2}=7.609 \times 10^{4} \mathrm{~m}^{-1}$.

In this simulation, the working gas is helium, which is modeled as a compressible ideal gas. Its viscosity is set to be temperaturedependent. The walls of WHX1 and WHX2 are maintained at $300 \mathrm{~K}$ while all the other system walls are assumed to be adiabatic. The initial temperature of the system is $300 \mathrm{~K}$. Each period of the piston movement is divided into 50 time steps. The convergence tolerance criterion for energy equations is $1 \times 10^{-6}$ and that for other equations is 0.001 . Running one case by a computer with 2.2 GHz processor and 2.0 GB RAM till steady state takes a month or even more.

In order to study the thermodynamic cycles of the working gas in refrigerator, some characteristic gas parcels are chosen. These

\section{WHX1 regenerator}

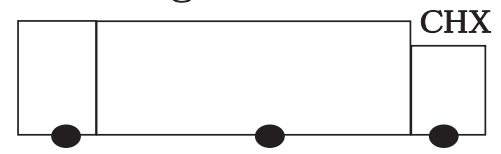

Fig. 2. Schematics of gas parcels for thermodynamic analysis.

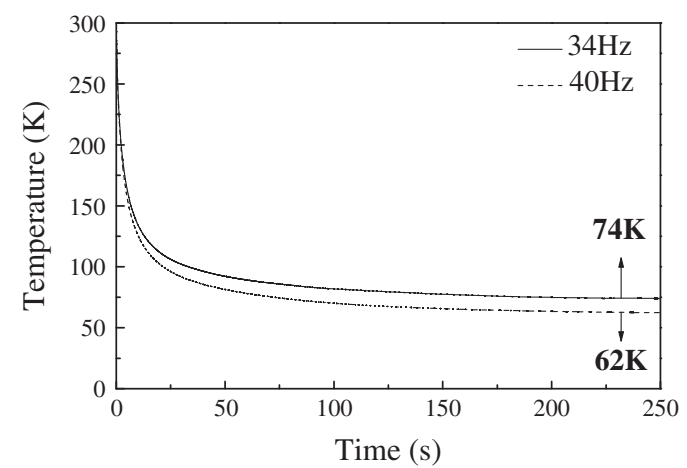

Fig. 3. Variations of cycle-average temperature of CHX wall surface for simulation cases. gas parcels are: gas parcel oscillating in after cooler (WHX1) and regenerator; gas parcel oscillating entirely in regenerator; and gas parcel oscillating in regenerator and CHX. The gas parcels chosen are located on the axis where the radial velocities of the gas parcels are far smaller than the axial velocities, so that the influences of the radial velocities of the gas parcels can be neglected. A schematic of gas parcels chosen for thermodynamic analysis is shown in Fig. 2.

Thermodynamic cycle of a gas parcel can be drawn on pressurespecific volume $(p-v)$ diagram and temperature-specific entropy $(T-s)$ diagram. The specific entropy change from the reference state of a gas parcel can be calculated as follows:

$s=C_{p} \ln \left(\frac{T}{T_{\text {ref }}}\right)+R \ln \left(\frac{p_{\text {ref }}}{p}\right)$

where $T_{\text {ref }}=288.15 \mathrm{~K}, p_{\text {ref }}=1.01325$ bar.

An additional Lagrangian method is used as a post-process to track the movement of a gas parcel to get its pressure $p$, specific volume $v$, temperature $T$ and axial velocity $v$ at different positions in a thermocycle. At time $t$, the pressure, specific volume and axial velocity of a chosen gas parcel are $p_{t}, v_{t}$ and $v_{t} . T_{t}$ and $s_{t}$ of the gas parcel can be obtained from ideal gas equation and Eq. (9). Assuming that the axial velocity of the gas parcel is keeping constant during a time step $\Delta t$ (if $\Delta t$ is small enough), therefore the gas parcel will move to a new certain position at time $t+\Delta t$. If the new position is not located in a grid point, $p_{t+\Delta t}, v_{t+\Delta t}$ and $v_{t+\Delta t}$ of the gas parcel are calculated by linear interpolation of those parameters at two adjacent grids. Again $T_{t+\Delta t}$ and $s_{t+\Delta t}$ of the gas parcel can be obtained from ideal gas equation and Eq. (9). After 50 time steps calculations, the gas parcel will return to its original position. Therefore thermodynamic cycles of the gas parcel can be obtained.

\section{Results and discussion}

\subsection{Refrigeration performance}

In this paper, the pulse tube refrigerator system has been simulated under two different operating frequencies, viz. the frequency of piston movement: $34 \mathrm{~Hz}$ and $40 \mathrm{~Hz}$. The frequency $34 \mathrm{~Hz}$ was in fact used in Havey's experiment [13]. The variations 


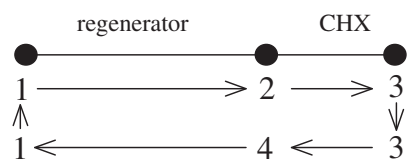

Fig. 5. A schematic of the working processes of a gas parcel oscillating in regenerator and $\mathrm{CHX}$ in a cycle.
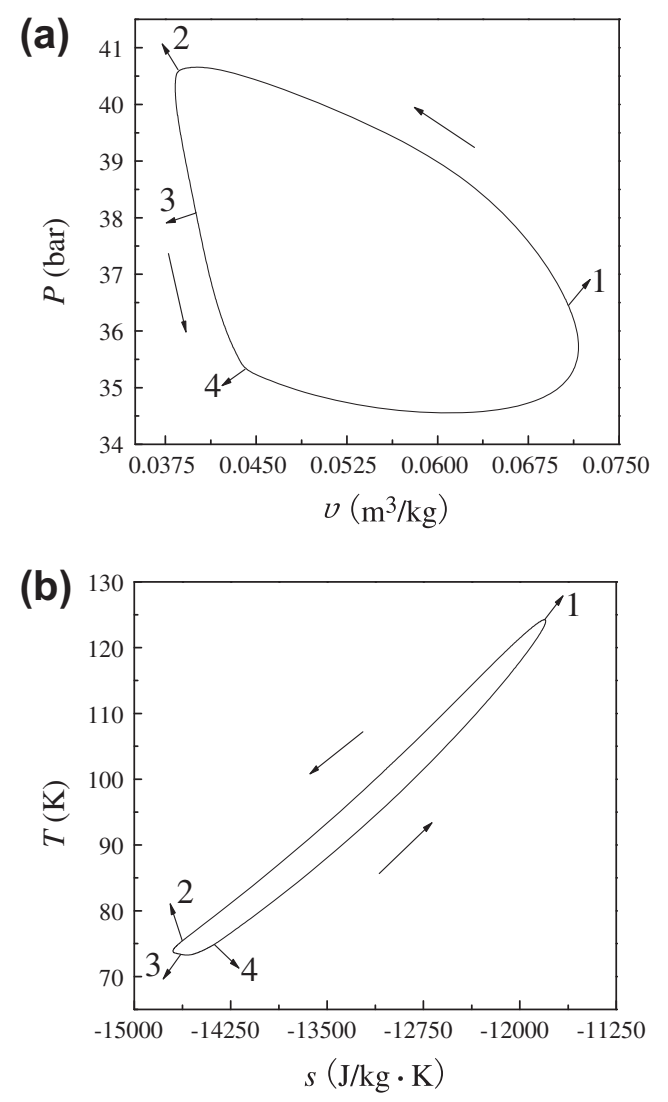

Fig. 6. (a) $p-v$ Diagram of the gas parcel oscillating in regenerator and CHX for the $34 \mathrm{~Hz}$ frequency case. (b) $T-s$ diagram of the gas parcel.

of cycle-average temperature of $\mathrm{CHX}$ wall surface are shown in Fig. 3 for the two cases. For $34 \mathrm{~Hz}$ frequency case, the simulated steady cycle-average temperature of CHX wall surface is $74 \mathrm{~K}$, which is lower than the experimental result $87 \mathrm{~K}$. The error between the simulation and experiment may be induced by the ideal gas assumption, the laminar flow assumption, or without wall thickness consideration. The $40 \mathrm{~Hz}$ frequency case has better performance than the $34 \mathrm{~Hz}$ frequency case, in which the simulated steady cycle-average CHX wall surface temperature is $62 \mathrm{~K}$.
Snapshot of the temperature contour along axial direction for 34 frequency case is shown in Fig. 4. It can be seen that the lowest temperature of the pulse tube refrigerator is mainly appearing in the CHX part.

\subsection{Thermodynamic cycles of gas parcels}

\subsubsection{Thermodynamic cycle of a gas parcel oscillating in regenerator and $\mathrm{CHX}$}

As discussed above, the lowest temperature is appearing in $\mathrm{CHX}$ part. However, the CHX is very short in length, and it is hard to find a gas parcel that only oscillates inner the CHX, so the gas parcel chosen is oscillating in both the regenerator and the $\mathrm{CHX}$. As shown in Fig. 5 , the cycle of the gas parcel is characterized by four points: point 1 is the leftmost position of the gas parcel in regenerator, point 3 is the rightmost position of the gas parcel in $\mathrm{CHX}$ (point 3 is in fact the outlet position of the CHX), points 2 and 4 correspond to the entrance position of the CHX.

The $p-v$ and $T-s$ diagrams of the gas parcel oscillating in regenerator and $\mathrm{CHX}$ for the $34 \mathrm{~Hz}$ frequency case are shown in Fig. 6a and $b$. The work and the heat transfer of the gas parcel during each cycle are listed in Tables 2 and 3. According to Fig. 6, the net effect of the cycle is consuming work and releasing heat. From point 1 to point 2 , the gas parcel experiences a compression process through regenerator. During processes $1-2$, the gas parcel releases heat, and the amount of heat out is larger than the amount of work in, so the temperature of the gas parcel decreases. During processes 2-3 within $\mathrm{CHX}$, the gas parcel produces work but also releases heat, the temperature of the gas parcel further decreases. During processes 3-4, the gas parcel produces work and absorbs heat, and the work produced by the gas parcel is lower than the heat absorbed by the gas parcel, so the temperature of the gas parcel increases. During processes $4-1$, the gas parcel continues to produce work and absorb heat, and also the amount of work out is lower than the amount of heat in, as a result the temperature of the gas parcel further increases to the original status. Moreover, it can be seen that the amount of heat in during processes $3-4$ is larger than the amount of heat out during processes 2-3 within $\mathrm{CHX}$. Thus the net effect of the gas parcel oscillating within CHX is absorbing heat and producing work. This explains why the lowest temperature is appearing in CHX. Noted that during the processes 1-2 and 4-1 within the regenerator, the net effect of the gas parcel is consuming work and releasing heat. And the amount of heat released by the gas parcel within regenerator is larger than the amount of heat absorbed within CHX. The difference exactly equals to the net work of the cycle required. So in a cycle, the gas parcel absorbs heat in CHX, consumes work and releases heat in regenerator like a heat pump.

All the illustrations above are for the refrigerator operating at $34 \mathrm{~Hz}$ frequency. The thermodynamic cycle of the gas parcel oscillating in regenerator and CHX for the $40 \mathrm{~Hz}$ frequency case is also shown in Fig. 7. Compared Figs. 6 and 7, it can be seen that

Table 2

Work of the gas parcel in each process and in a cycle.

\begin{tabular}{|c|c|c|c|c|c|c|}
\hline$W_{1-2}(\mathrm{~J} / \mathrm{kg})$ & $W_{2-3}(\mathrm{~J} / \mathrm{kg})$ & $W_{3-4}(\mathrm{~J} / \mathrm{kg})$ & $W_{4-1}(\mathrm{~J} / \mathrm{kg})$ & $W_{1-2}+W_{4-1}(\mathrm{~J} / \mathrm{kg})$ & $W_{2-3}+W_{3-4}(\mathrm{~J} / \mathrm{kg})$ & $W_{12341}(\mathrm{~J} / \mathrm{kg})$ \\
\hline-127327.2 & 5729.9 & 14661.3 & 93370.3 & -33956.9 & 20391.2 & -13565.7 \\
\hline
\end{tabular}

Table 3

Heat transfer of the gas parcel in each process and in a cycle.

\begin{tabular}{|c|c|c|c|c|c|c|}
\hline$q_{1-2}(\mathrm{~J} / \mathrm{kg})$ & $q_{2-3}(\mathrm{~J} / \mathrm{kg})$ & $q_{3-4}(\mathrm{~J} / \mathrm{kg})$ & $q_{4-1}(\mathrm{~J} / \mathrm{kg})$ & $q_{1-2}+q_{4-1}(\mathrm{~J} / \mathrm{kg})$ & $q_{2-3}+q_{3-4}(\mathrm{~J} / \mathrm{kg})$ & $q_{1234}(\mathrm{~J} / \mathrm{kg})$ \\
\hline-280072.6 & -382.6 & 19284.9 & 247604.6 & -32468 & 18902.3 & -13565.7 \\
\hline
\end{tabular}



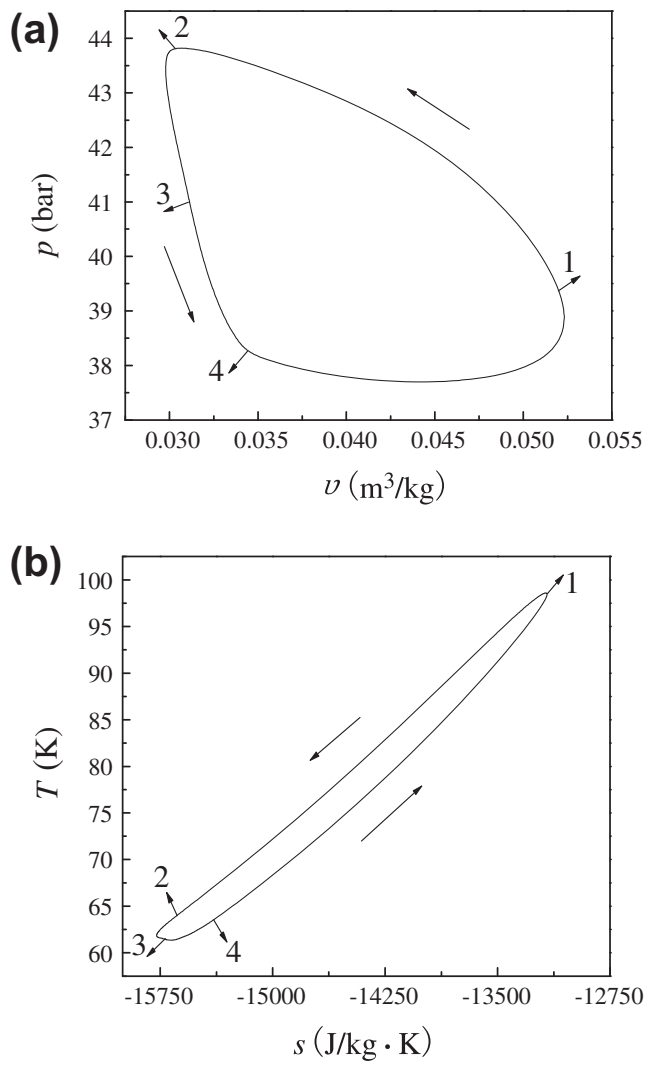

Fig. 7. (a) $p-v$ Diagram of the gas parcel oscillating in regenerator and CHX for the $40 \mathrm{~Hz}$ frequency case. (b) $T-s$ diagram of the gas parcel.

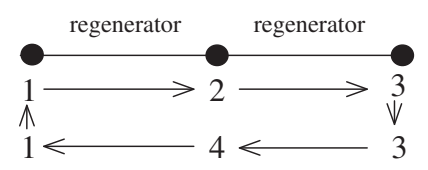

Fig. 8. A schematic of the working processes of a gas parcel oscillating in regenerator in a cycle.

thermodynamic cycle of the gas parcel oscillating in regenerator and $\mathrm{CHX}$ for the $40 \mathrm{~Hz}$ frequency case are very similar to that for the $34 \mathrm{~Hz}$ frequency case. The results indicate that at the same location the working gases of different frequencies undergo the same type of thermodynamic cycles.

3.2.2. Thermodynamic cycles of gas parcels oscillating in regenerator

From Fig. 8, it can be observed that the cycle of a gas parcel oscillating in regenerator is characterized by four points: point 1
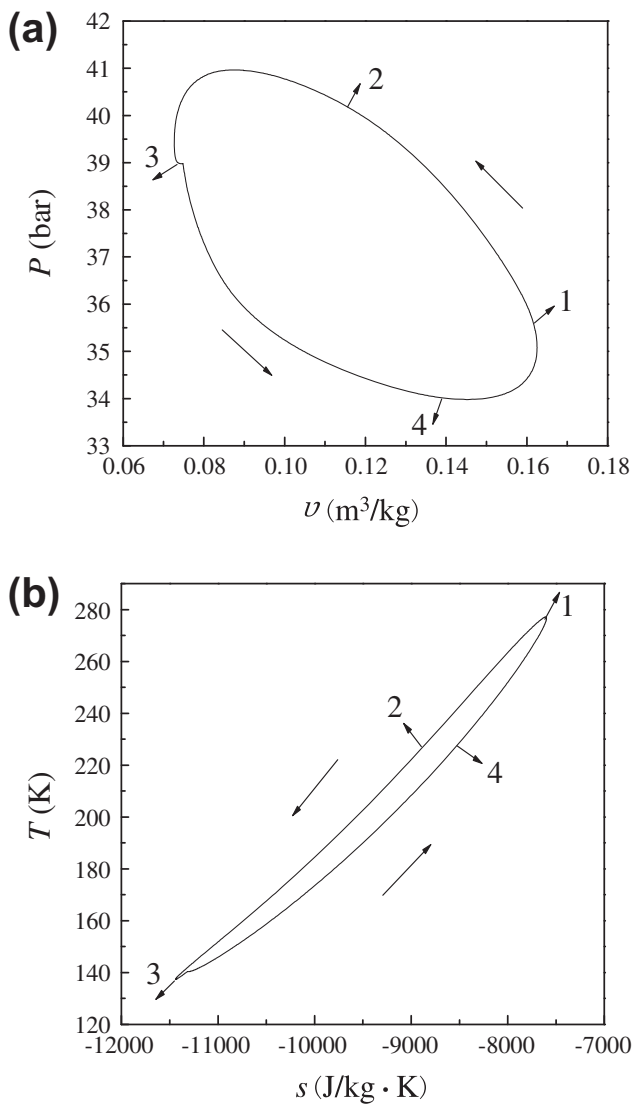

Fig. 9. (a) $p-v$ Diagram of the gas parcel oscillating in regenerator for the $34 \mathrm{~Hz}$ frequency case. (b) $T-s$ diagram of the gas parcel.

is the leftmost position, point 3 is the rightmost position, points 2 and 4 are the central position of the regenerator.

Fig. 9a shows the $p-v$ diagram of the gas parcel oscillating in regenerator for the $34 \mathrm{~Hz}$ frequency case, and Fig. 9b shows the $T-s$ diagram of the gas parcel. The work and heat transfer of the gas parcel are listed in Tables 4 and 5.

Clearly, the gas parcel oscillating in regenerator also undergoes a cycle consuming work and releasing heat. During the processes $1-2$ and $2-3$, the gas parcel consumes work and releases heat, while during the processes 3-4 and $4-1$, the gas parcel produces work and absorbs heat. During processes $2-3-4$, the net effect of the gas parcel is absorbing heat and producing work in the low temperature part. While during the processes $4-1-2$, the net effect of the gas parcel is consuming work and releasing heat in the high temperature part. And again the amount of heat released in the high temperature part of the regenerator is larger than the heat absorbed in the low temperature part of the regenerator, and the difference equals to the

Table 4

Work of the gas parcel in each process and in a cycle.

\begin{tabular}{|c|c|c|c|c|c|c|}
\hline$W_{1-2}(\mathrm{~J} / \mathrm{kg})$ & $W_{2-3}(\mathrm{~J} / \mathrm{kg})$ & $W_{3-4}(\mathrm{~J} / \mathrm{kg})$ & $W_{4-1}(\mathrm{~J} / \mathrm{kg})$ & $W_{1-2}+W_{4-1}(\mathrm{~J} / \mathrm{kg})$ & $W_{2-3}+W_{3-4}(\mathrm{~J} / \mathrm{kg})$ & $W_{12341}(\mathrm{~J} / \mathrm{kg})$ \\
\hline-184788.7 & -155759.5 & 222361.8 & 78563.1 & -106225.6 & 66602.3 & -39623.3 \\
\hline
\end{tabular}

Table 5

Heat transfer of the gas parcel in each process and in a cycle.

\begin{tabular}{|c|c|c|c|c|c|c|}
\hline$q_{1-2}(\mathrm{~J} / \mathrm{kg})$ & $q_{2-3}(\mathrm{~J} / \mathrm{kg})$ & $q_{3-4}(\mathrm{~J} / \mathrm{kg})$ & $q_{4-1}(\mathrm{~J} / \mathrm{kg})$ & $q_{1-2}+q_{4-1}(\mathrm{~J} / \mathrm{kg})$ & $q_{2-3}+q_{3-4}(\mathrm{~J} / \mathrm{kg})$ & $q_{1234}(\mathrm{~J} / \mathrm{kg})$ \\
\hline-361683.4 & -405817.1 & 493270.5 & 234606.7 & -127076.7 & 87453.4 & -39623.3 \\
\hline
\end{tabular}


net work of the gas parcel in a cycle. So the gas parcel also consumes work and pumps heat from the right low temperature part to the left high temperature part of the regenerator. The

(a)

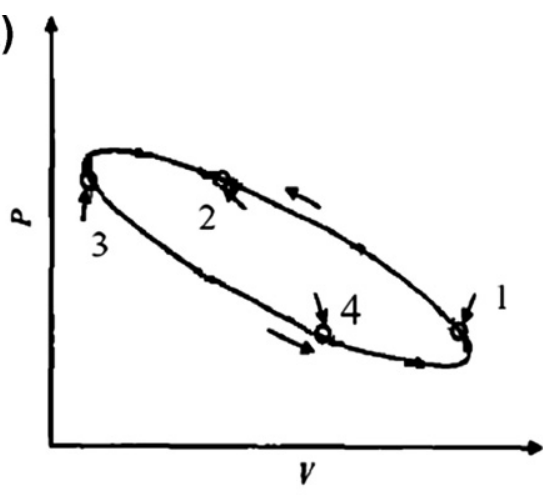

(b)

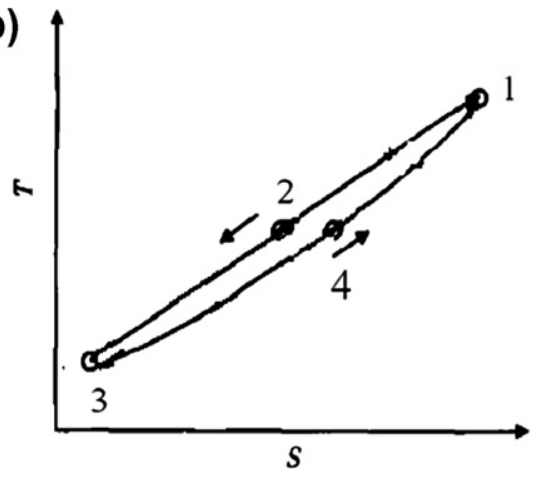

Fig. 10. (a) $p-v$ Diagram of the gas parcel oscillating in regenerator theoretical attained by Luo [11]. (b) T-s diagram of the gas parcel.
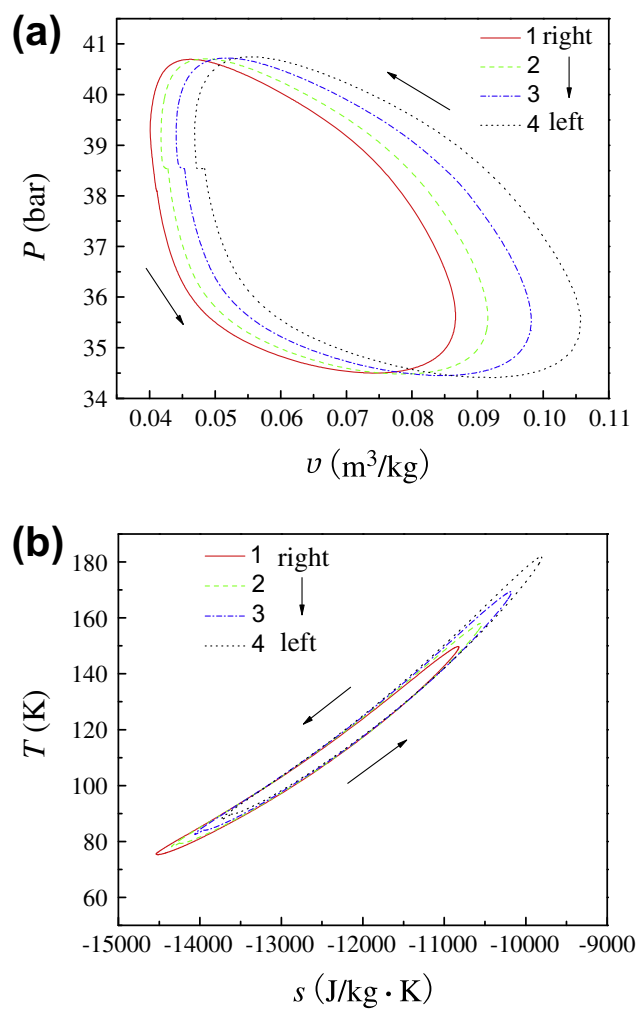

Fig. 11. (a) $p-v$ Diagrams of the gas parcels oscillating in regenerator for $34 \mathrm{~Hz}$ frequency case. (b) $T-s$ diagrams of those gas parcels. way of heat transfer within the regenerator is very similar with that between $\mathrm{CHX}$ and regenerator. It should be noted that thermodynamic cycle of the gas parcel oscillating in regenerator of $40 \mathrm{~Hz}$ frequency case is also very similar with that of the $34 \mathrm{~Hz}$ frequency case.

Fig. 10 is the thermodynamic cycle of a gas parcel oscillating in a regenerator attained by Luo [11] from pure thermodynamic analysis. The four points characterized the thermodynamic cycles in Fig. 10a and b have the same meaning with those in Figs. 8-10. It can be seen that thermodynamic cycle of the gas parcel oscillating in regenerator is quite similar between simulation and theoretical analysis. But the later one is only qualitative.

Fig. 11a and b shows the $p-v$ diagrams and $T-s$ diagrams of four adjacent gas parcels oscillating entirely in regenerator. Gas parcel 1 lies in the rightmost while gas parcel 4 lies in the leftmost. From Fig. 11, it can be seen that each gas parcel in regenerator undergoes the same type of thermodynamic cycle but at different temperatures. And all of the gas parcels in regenerator work together to pump heat from the low temperature part to the high temperature part of the regenerator.

\subsubsection{Thermodynamic cycle of a gas parcel oscillating in WHX1 and regenerator}

As shown in Fig. 12, the cycle of the gas parcel oscillating in WHX1 and regenerator is also characterized by four points: point

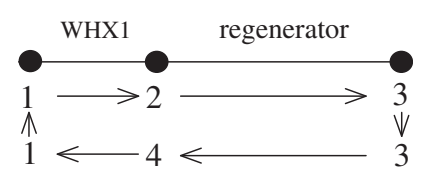

Fig. 12. A schematic of the working processes of a gas parcel oscillating in WHX1 and regenerator in a cycle.
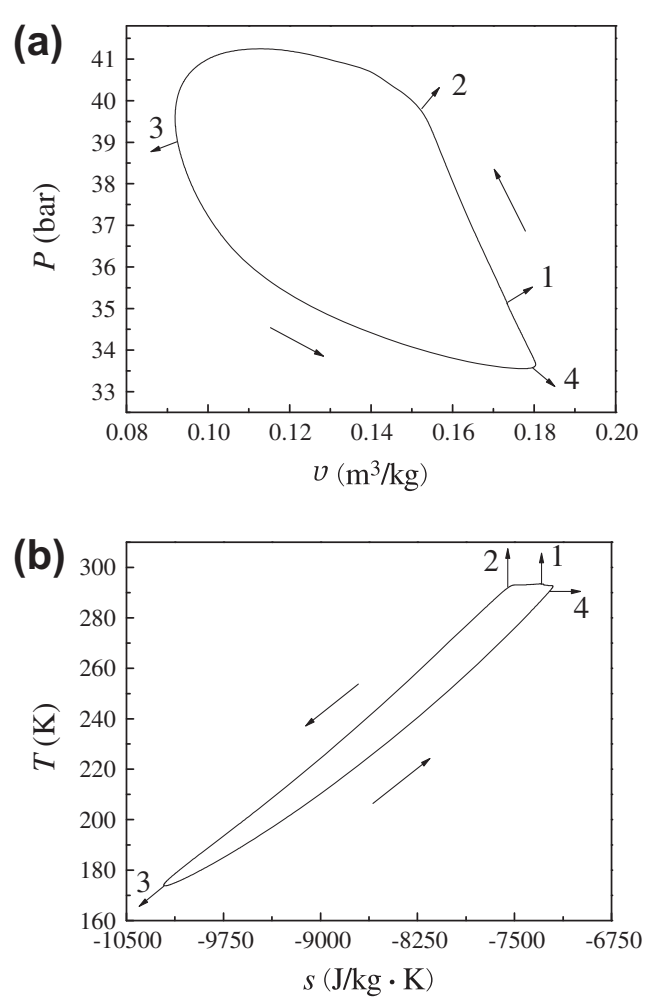

Fig. 13. (a) $p-v$ Diagram of the gas parcel oscillating in WHX1 and regenerator for the $34 \mathrm{~Hz}$ frequency case. (b) $T-s$ diagram of the gas parcel. 
Table 6

Work of the gas parcel in each process and in a cycle.

\begin{tabular}{|c|c|c|c|c|c|c|}
\hline$W_{1-2}(\mathrm{~J} / \mathrm{kg})$ & $W_{2-3}(\mathrm{~J} / \mathrm{kg})$ & $W_{3-4}(\mathrm{~J} / \mathrm{kg})$ & $W_{4-1}(\mathrm{~J} / \mathrm{kg})$ & $W_{1-2}+W_{4-1}(\mathrm{~J} / \mathrm{kg})$ & $W_{2-3}+W_{3-4}(\mathrm{~J} / \mathrm{kg})$ & $W_{12341}(\mathrm{~J} / \mathrm{kg})$ \\
\hline-77670.8 & -245901.9 & 299695.8 & -16603.9 & -94274.7 & 53793.9 & -40480.8 \\
\hline
\end{tabular}

Table 7

Heat transfer of the gas parcel in each process and in a cycle.

\begin{tabular}{|c|c|c|c|c|c|c|}
\hline$q_{1-2}(\mathrm{~J} / \mathrm{kg})$ & $q_{2-3}(\mathrm{~J} / \mathrm{kg})$ & $q_{3-4}(\mathrm{~J} / \mathrm{kg})$ & $q_{4-1}(\mathrm{~J} / \mathrm{kg})$ & $q_{1-2}+q_{4-1}(\mathrm{~J} / \mathrm{kg})$ & $q_{2-3}+q_{3-4}(\mathrm{~J} / \mathrm{kg})$ & $q_{1234}(\mathrm{~J} / \mathrm{kg})$ \\
\hline-81013.3 & -615532.2 & 677157.3 & -21092.6 & -102105.9 & 61625.1 & -40480.8 \\
\hline
\end{tabular}

1 is the leftmost position in WHX1, point 3 is the rightmost position in regenerator, and points 2 and 4 locate on the entrance position of the regenerator. The $p-v$ diagram and $T-s$ diagram of the gas parcel are shown in Fig. 13a and b. The work and heat transfer of the gas parcel are listed in Tables 6 and 7.

It can be seen that when the gas parcel oscillates in regenerator part, it absorbs heat and produces work, and when it oscillates in WHX1 part, it consumes work and releases heat. Over all, the gas parcel oscillating in WHX1 and regenerator also consumes work and pumps heat from the low temperature part (right in regenerator) to the high temperature part (left in WHX1). The heat released in WHX1 by the gas parcel will be ultimately conveyed to the WHX1 wall surface, where the temperature is maintaining at $300 \mathrm{~K}$. Again, thermodynamic cycle of the gas parcel oscillating in WHX1 and regenerator for $40 \mathrm{~Hz}$ frequency is similar to that for $34 \mathrm{~Hz}$ frequency case.

\section{Conclusions}

In this paper an inertance tube pulse tube refrigerator is simulated by a two-dimensional axial-symmetric CFD model. The simulation results are summarized in Lagrangian's view and the thermodynamic cycles of gas parcels are obtained. Results show that different gas parcels undergo thermodynamic cycles at different temperatures. These gas parcels convey heat from the low temperature part to the high temperature part of the system. Finally, the heat will be released from the after cooler wall surface, where the temperature is keep at $300 \mathrm{~K}$. Results of different operating frequency cases indicate that the working gas parcels at the same part of the system but under different working conditions might undergo the same type of thermodynamic cycles. The thermodynamic cycles in the regenerator are summarized from CFD calculations, and are compared with the thermodynamic analysis result from a reference. Comparison shows that both CFD simulation and theoretical analysis can predict the similar thermodynamic cycles, but only CFD simulation gives the quantitative results.

\section{Acknowledgement}

This work is supported by the National Science Foundation of China under Contract No. 50890182.

\section{References}

[1] Gifford W, Longsworth R. Pulse tube refrigeration. Trans ASME, J Eng Ind (Ser B) $1964 ; 86: 264-8$.

[2] Mikulin EA, Tarasov AA, Shkrebyonock MP. Low-temperature expansion pulse tubes. Adv Cryo Eng 1984;29:629-37.

[3] Zhu S, Wu P, Chen Z. A single stage double inlet pulse tube refrigerator capable of reaching $42 \mathrm{~K}$. In: ICEC 13 proc cryogenics, Beijing, vol. 30; 1990. p. 256-61.

[4] Cai J, Wang J, Zhou Y. Experimental analysis of double-inlet principle in pulse tube refrigerator. Cryogenics 1993;33:522-5.

[5] Kanao K, Watanabe N, Kanazawa Y. A miniature pulse tube refrigerator for temperature below $100 \mathrm{~K}$. Cryogenics 1994;34(suppl. 1):167-70.

[6] Jiang N. A $3 \mathrm{He}$ pulse tube cooler operating down to $1.3 \mathrm{~K}$. Cryogenics 2004;44(11):809-16.

[7] Gifford W, Longsworth R. Surface heat pumping. Adv Cryo Eng 1966;11:171-81.

[8] Peter S, Radebaugh R. Development and experimental test of an analysis model of the orifice pulse tube refrigerator. Adv Cryo Eng 1987;33:851-9.

[9] Liang J, Ravex A, Rolland P. Study on pulse tube refrigeration part 1: thermodynamic non-symmetry effect. Cryogenics 1996;36(2):87-93.

[10] Liang J. Thermodynamic cycles in oscillating flow regenerators. J Appl Phys 1997;82(9):4159-63.

[11] Luo E, Dai W, Wu Z, et al. Meso-scope thermodynamic theory for cyclic flow engines. Cryogenics 2004;1:1-11 [in Chinese].

[12] Cha J, Ghiaasiaan S, Desai P, et al. Multi-dimensional flow effects in pulse tube refrigerators. Cryogenics 2006;46:658-65.

[13] Harvey J. Oscillatory compressible flow and heat transfer in porous media application to cryocooler. PhD thesis. Georgia Institute of Technology, Atlanta; 2003.

[14] Fluent INC. Fluent 6 user manual. Fluent INC; 2003. 